\title{
Impacto na qualidade de vida de pacientes submetidos a otoplastia em um hospital universitário
}

\section{Impact of otoplasty on patient's quality of life in a university hospital}

Monique Barros Brito da Conceição ${ }^{1}$. Anna Caroline Rodrigues de Souza Matos ${ }^{1}$. Clara Mota Randal Pompeu de Almeida ${ }^{1}$. Antonio Brazil Viana Júnior ${ }^{1}$. Viviane Carvalho da Silva ${ }^{1,2}$. Rodolfo Borsaro Bueno Jorge ${ }^{1,2}$. Aline Almeida Figueiredo Borsaro ${ }^{1,2}$.

1 Universidade Federal do Ceará (UFC), Fortaleza, Ceará, Brasil. 2 Hospital Universitário Walter Cantídio (HUWC), Fortaleza, Ceará, Brasil.

\section{RESUMO}

Objetivos: analisar o impacto na qualidade de vida dos pacientes com orelhas proeminentes submetidos a otoplastia. Metodologia: foram selecionados 27 pacientes maiores de 15 anos. No pré-operatório: aplicado Escala Visual Analógica (EVA) para satisfação de aparência física. Após 2 meses: EVA e Escala de Resultados de Glasgow (ERG) para avaliar qualidade de vida pós-cirúrgica. Foi definido um Delta EVA (diferença entre pós e pré-operatório), relacionando-o com a ERG. Resultados: a mediana da EVA préoperatória foi 50 e a pós-operatória, 100 . Na ERG, a mediana da pontuação total foi 52,78 (saúde geral=75; relações sociais=16,67; saúde física=zero). Na análise de Delta EVA com ERG, o coeficiente de correlação de Spearman foi: pontuação total=0,599 $(p=0,001)$, saúde geral=0,571 $(p=0,002)$ e relações sociais=0,445 $(p=0,02)$, evidenciando correlação positiva significativa. Conclusão: a Otoplastia mostrou-se importante na melhora da qualidade de vida de pacientes com orelhas proeminentes, principalmente na saúde geral e relações sociais. Comparando e analisando os resultados, foi possível concluir que a melhora na satisfação com a aparência física é fator preditor direto de mudança na qualidade de vida.

Palavras-chave: Orelha. Pavilhão auricular. Qualidade de vida. Cirurgia.

\section{ABSTRACT}

Objectives: To analyze the impact on the quality of life of patients with prominent ears that underwent otoplasty. Methods: 27 patients over 15 years old were included. During the preoperative evaluation a Visual Analogue Scale (VAS) was applied in order to measure satisfaction with physical appearance. After two months postoperative VAS and Glasgow Benefit Inventory (GBI) were used to evaluate patients' quality of life. Furthermore, a delta-VAS was established before and after surgery, relating to the GBI. Results: The preoperative VAS median was 50 and the postoperative was 100. GBI total median was 52.78 (general health 75 ; social relations 16.67; physical health 0). Regarding the Delta-VAS analysis with GBI, the Spearman correlation coefficient was: total results $0.99(\mathrm{p}=0.001)$, general health $0.571(\mathrm{p}=0.002)$ and social relations $0.445(\mathrm{p}=0.02)$, presenting significant positive effects. Conclusion: The otoplasty led to improvement in the patients' quality of life with prominent ears, mostly in general health and social relations. Comparing and analyzing the results, it is possible to conclude that the improvement in satisfaction with physical appearance is directly predicting change in quality of life.

Keywords: Ear. Ear auricle. Quality of life. Surgery.

Autor correspondente: Monique Barros Brito da Conceição, Rua Capitão Francisco Pedro, 1290, Rodolfo Teófilo, Fortaleza, Ceará. CEP: 60430372. Telefone: +55 85 98823-2729. E-mail: moniquebarrrosb@gmail.com

Conflito de interesses: Não há qualquer conflito de interesses por parte de qualquer um dos autores.

Recebido em: 07 Set 2020; Revisado em: 01 Mar 2021; Aceito em: 02 Mar 2021. 


\section{INTRODUÇÃO}

A Organização Mundial de Saúde (OMS) definiu como Qualidade de Vida (QV) a "percepção do indivíduo sobre a sua posição na vida, dentro do contexto dos sistemas de cultura e valores nos quais está inserido e em relação aos seus objetivos, expectativas, padrões e preocupações". ${ }^{1}$ Tal definição reflete uma avaliação subjetiva, contemplando a influência da saúde psicológica e física, assim como das relações sociais, crenças e nível de independência. ${ }^{2}$

Recentemente, vem surgindo uma preocupação maior quanto à avaliação do impacto das doenças no comprometimento das atividades diárias, assim como na percepção de saúde do paciente. Segundo a OMS, a definição de saúde, além de ausência de enfermidades, também envolve o bem-estar físico, mental e social. Dessa forma, a qualidade de vida e saúde estão diretamente ligadas. ${ }^{3}$

Orelhas proeminentes é uma deformidade congênita da orelha externa, transmitida de forma autossômica dominante. É uma das mais comuns deformidades da orelha externa, representando $5 \%$ da população geral. ${ }^{4,5}$ Seu desenvolvimento é devido duas principais alterações anatômicas: o excesso de concha e/ou a dobra da anti-hélice ausente ou subdesenvolvida. ${ }^{6}$

Pacientes com orelhas proeminentes são passíveis de desenvolverem problemas psicológicos, tais como ansiedade, baixa autoestima e isolamento social. Esses distúrbios levam a um estresse emocional e, consequentemente, a um declínio na qualidade de vida e interferência negativa no desempenho na escola ou trabalho. ${ }^{6}$ Pacientes com tal deformidade, muitas vezes, são submetidos a ridicularização, resultando em diversos distúrbios emocionais. ${ }^{5,7}$

A otoplastia é um procedimento comum para a correção das orelhas proeminentes. O objetivo principal do tratamento é posicionar a orelha com boa simetria e boa forma. ${ }^{4}$ Diversas técnicas cirúrgicas foram propostas até o momento. Dentre elas, encontra-se a técnica eclética, que é derivada das contribuições de diversos autores, tais como Mustadé ${ }^{8}$ e Furnas, ${ }^{9}$ com modificações pessoais, que objetivaram um procedimento mais rápido, seguro e eficiente. ${ }^{10,11} \mathrm{Um}$ dos principais motivos para se realizar a otoplastia é a insatisfação estética. A associação entre a correção cirúrgica e a redução do sofrimento psicológico e melhora da autoestima já está bem documentada. ${ }^{12,5,7}$

Atualmente, cresce a busca por métodos de pesquisa que avaliem a satisfação e alterações da qualidade de vida dos pacientes frente aos tratamentos submetidos. Um dos objetivos para tal crescimento é a avaliação para adoção de novas terapias ou consolidação das terapias clássicas. ${ }^{7}$

$\mathrm{Na}$ literatura atual, há uma relativa escassez de dados que avaliam o impacto da otoplastia na qualidade de vida dos pacientes. A Escala de Resultados de Glasgow (ERG) é um questionário que avalia a qualidade de vida relacionada à saúde, que foi criado para procedimentos e intervenções otorrinolaringológicas..$^{13,14,15} \mathrm{O}$ uso de inquéritos validados, como exemplo a ERG, auxilia na obtenção dos dados, permitindo, assim, o fortalecimento da importância da otoplastia no nosso meio.?

Sendo a orelha de abano uma condição bastante comum, que leva a problemas sociais e psicológicos importantes, faz-se necessário maiores estudos para avaliar se há melhoria da qualidade de vida frente ao procedimento cirúrgico para correção da deformidade. Dessa forma, poderá se chamar a atenção para maiores investimentos em otoplastia, assim como ratificar a importância desse procedimento cirúrgico.

O objetivo geral do estudo é analisar o impacto na qualidade de vida dos pacientes com orelha proeminentes submetidos a otoplastia. Assim como avaliar a modificação da saúde geral, física e social nesses pacientes; comparar a satisfação da aparência física no pré e no pós-operatório; comparar a variação dos valores da EVA pré e pós-operatória com o resultado da ERG; e verificar se há correlação entre a idade dos pacientes e os resultados da ERG.

\section{MÉTODOS}

O presente estudo observacional foi aprovado pelo Comitê de Ética em Pesquisa (CEP), com o número de protocolo sendo 2.834.563. Os pacientes receberam explicações a respeito da pesquisa e, ao concordarem em participar, assinaram o Termo de Consentimento Livre e Esclarecido (TCLE).

A pesquisa foi realizada no serviço de Otorrinolaringologia de um hospital universitário. As otoplastias foram realizadas no ambulatório de pequenas cirurgias. Foram selecionados, em uma amostra consecutiva, 27 pacientes, maiores de 15 anos, dentre homens e mulheres, atendidos no ambulatório de Plástica Facial, com queixa de "orelhas proeminentes" no período de agosto de 2018 a julho de 2019.

Para exclusão do estudo, foram utilizados os seguintes critérios: recusa em participar da pesquisa e em assinar o TCLE, incapacidade de ler ou responder o questionário, otoplastia revisional, deformidade unilateral e alteração lobular isolada, microtia e alterações genéticas, incluindo síndrome craniofacial, e pacientes portadores de comorbidades incompatíveis com o ato cirúrgico.

Aplicaram-se os seguintes questionários: Escala Visual Analógica (EVA) sobre a aparência estética e Escala de Resultados de Glasgow (ERG). Foram utilizados em dois momentos: no pré-operatório, foi respondido a EVA e, no pós-operatório, a ERG e a EVA.

A Escala de Resultados de Glasgow (ERG) visa avaliar a qualidade de vida pós-cirúrgica. Baseia-se em 18 questões distribuídas em três grupos: doze em saúde geral, três em saúde física e três em saúde social. A cada pergunta, são possíveis cinco respostas, com uma pontuação variando de 
1 a 5. A pontuação final será distribuída entre -100 e +100 (do pior ao melhor), calculada através da seguinte fórmula matemática: a pontuação total é dividida pelo número de perguntas, subtraindo 3 e multiplicando por $50 .{ }^{16}$

A Escala Visual Analógica (EVA) foi utilizada para medir o julgamento subjetivo em relação ao grau de satisfação da aparência estética, comparando o antes e o depois da otoplastia, variando a pontuação de 0 a 100, sendo zero a menor satisfação e cem a maior possível. Foi utilizado também como variável o valor de Delta EVA, que representa a diferença entre o valor de EVA pós-operatório e pré-operatório.

As cirurgias foram realizadas pelos residentes do terceiro ano (R3), sob a supervisão do médico responsável pelo ambulatório de plástica facial do serviço de otorrinolaringologia, utilizando a técnica cirúrgica eclética, descrita abaixo. ${ }^{12}$

Realizada anestesia local com infiltração de xilocaína e adrenalina (1:200.000). Em seguida, incisão da pele na face medial do pavilhão, em forma navicular, com cerca de $1,5 \mathrm{~cm}$ de largura, seguida de descolamento da pele até a borda posterior da hélice e região da mastoide. Realizada ressecção do excesso de concha. Em seguida, marcação da dobra da anti-hélice e suturas de Mustardè, transfixando a cartilagem mediolateralmente, sem ultrapassar a pele da face lateral, com fio mononylon incolor 4.0. Logo após, fixação da concha no periósteo da mastoide com mononylon preto 3.0, retroposicionando-se o pavilhão. Caso seja necessário, pode ser realizada a correção do lóbulo lateralizado. Por fim, sutura simples da incisão, com nylon 5.0, e curativo compressivo.

Para análise estatística, utilizou-se os programas JAMOVI 1.2.2. Os dados do estudo foram coletados e gerenciados usando a ferramenta de eletrônica de coleta e gerenciamento de dados REDCap. ${ }^{17}$

\section{RESULTADOS}

De 34 pacientes que realizaram a otoplastia, 27 responderam a todos os questionários. Desse total, $51,9 \%$ eram do sexo masculino e $48,1 \%$ do sexo feminino. A mediana de idade foi de 27,67 anos, sendo a mínima de 15 anos e a máxima de 47 anos.

Na Escala Visual Analógica (EVA) pré-operatória, a mediana foi de 50 , sendo o $25^{\circ}$ percentil (p-25) igual a 20 e o $75^{\circ}$ percentil (p-75) igual a 60. A nota mínima antes da cirurgia foi de zero e a máxima de 80 . Na avaliação pós-operatória pela EVA, a mediana foi de 100 , com p-25 de 90 e p-75 de 100, sendo a nota mínima 70 e a máxima 100 (Figura 1). Foi evidenciado melhora estatisticamente significativa entre a pontuação total avaliada no pré-operatório e pós-operatório, com $\mathrm{p}<0,001$.

O Delta EVA obteve uma mediana de 45, sendo a variação máxima de 100 e a mínima de 10. Dos 27 pacientes, 8 tiveram delta EVA igual a 40 e 5 pacientes igual a 80 . (Figura 2). Nos pacientes do sexo masculino, a mediana foi de 42,5, sendo p-25 de 40 e p-75 de 60, com mínimo de
10 e máximo de 80 . Nos femininos, mediana de 50, p-25 de 40, p-75 de 80, com mínimo de 25 e máximo de 100 . Não houve diferença significativa entre os $\operatorname{sexos}(\mathrm{p}=0,28)$. Ao relacionar com a idade através do $\rho$ de Spearman, não foi obtido correção significativa (coeficiente $=0,347$ e $\mathrm{p}=$ 0,077).

Figura 1. Análise da Escala Visual Analógica - *p<0,001.

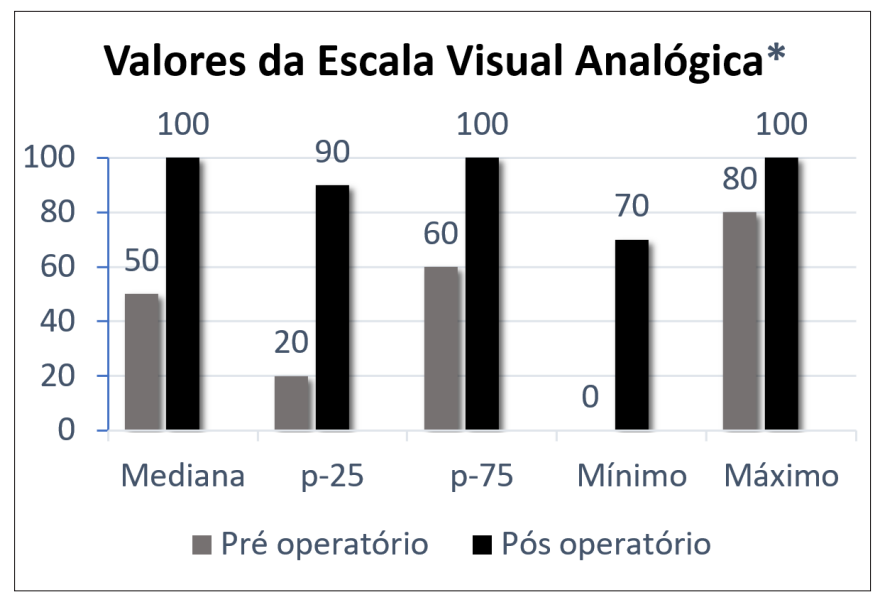

Figura 2. Número de pacientes para cada valor de Delta EVA.

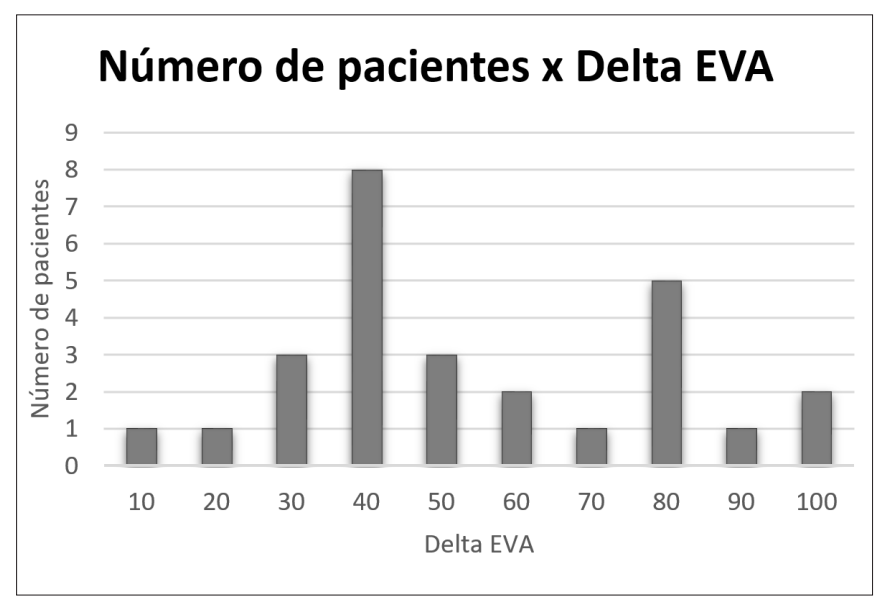

Na Escala de Resultados de Glasgow (ERG), a mediana da pontuação total foi 52,78 (55,56 para as mulheres e 51,39 para os homens), sendo 75 na categoria saúde geral (homens $=68,75$, mulheres $=75), 16,67$ em relações sociais (sexo feminino $=$ 33,33 , sexo masculino $=16,67$ ) e zero em saúde física para ambos os sexos. Não houve diferença significativa entre os sexos $(\mathrm{p}=0,83)$. A pontuação máxima total foi de 86,11 e a mínima de 2,78. Em relação à saúde geral, a máxima foi de 100 e a mínima de 0 . Na categoria relações sociais, a mínima foi zero e a máxima 100. Já na avaliação da saúde física, a mínima foi -16,67 e a máxima 100, tendo 4 pacientes apresentado valor negativo e 14 pacientes valor zero (Tabela 1 ). Ao correlacionar com a idade, obtivemos um coeficiente $\rho$ de Spearman para a pontuação total de 0,437 , com $p=0,022$, demonstrando uma relação significativa positiva no nível de 0,05 . 
Na análise de Delta EVA e a Escala de Resultado de Glasgow, o coeficiente de correlação de Spearman foram os seguintes: pontuação total $=0,599(\mathrm{p}=0,001)$, saúde geral $=0,571(\mathrm{p}=$ $0,002)$, para relações sociais $=0,445(\mathrm{p}=0,02)$, evidenciando correlação positiva significativa entre as variáveis. Para a relação com a pontuação da saúde física, o $\rho$ de Spearman foi de 0,217 $(\mathrm{p}=0,277)$, não apresentando correlação significativa (Tabela 2).
Utilizando a idade como variável de controle na correlação parcial entre Delta EVA e ERG, a correlação significativa positiva para pontuação total (coeficiente $=0,557$ e $\mathrm{p}=0,003$ ), saúde geral (coeficiente $=0,518$ e $\mathrm{p}=0,007$ ) e relações sociais (coeficiente $=0,554$ e $p=0,003$ ) se mantem. Delta EVA e saúde física permanecem sem correlação significativa (coeficiente $=-0,14$ e $\mathrm{p}=0,945)$.

Tabela 1. Análise da pontuação total e da pontuação de saúde geral, relações sociais e saúde física da ERG.

\begin{tabular}{ccccc}
\hline & Pontuação total & Saúde geral & Relações sociais & Saúde física \\
\hline Mediana & 52,78 & 75 & 16,67 & 0 \\
Máxima & 86,11 & 100 & 100 & 100 \\
Mínima & 2,78 & 0 & 0 & $-16,67$ \\
\hline
\end{tabular}

Tabela 2. Coeficiente de correlação de Spearman entre o Delta EVA e a Escala de Resultado de Glasgow.

\begin{tabular}{ccccc}
\hline & Pontuação total & Saúde geral & Relações sociais & Saúde Física \\
\hline $\begin{array}{c}\text { Coeficiente de } \\
\text { correlação } \\
\text { Valor p }\end{array}$ & 0,599 & 0,571 & 0,445 & 0,217 \\
\hline
\end{tabular}

\section{DISCUSSÃO}

Pacientes com orelhas proeminentes podem apresentar alterações psicológicas devido às experiências psicossociais negativas sofridas e ao descontentamento com a aparência física, gerando cada vez mais dificuldade em realizar suas atividades do cotidiano, tais como na vida pessoal e profissional. Nesse estudo, foi possível avaliar o impacto da otoplastia na vida desses pacientes.

Através da Escala Visual Analógica (EVA) avaliada em dois momentos, pré e pós-operatório, foi possível comparar o grau de satisfação com a aparência física dos pacientes com suas orelhas antes e depois da cirurgia. Foi evidenciado uma melhora significativa após o procedimento cirúrgico $(p<0,001)$. Em nenhuma das respostas houve decréscimo na nota. Todos obtiveram resultados positivos na variável Delta EVA.

Dentro da análise da Escala de Resultados de Glasgow, houve resultados positivos em quase todos os aspectos do questionário, dentre eles a pontuação total (mediana de 52,58), a de saúde geral (75) e a de relações sociais $(16,67)$, mostrando uma melhora na qualidade de vida após a otoplastia desses pacientes, conforme mostra Choi et al, ${ }^{6}$ Braun et al ${ }^{18}$ e Maroni et al. ${ }^{4}$

Braun et al, ${ }^{18}$ analisando 21 pacientes adultos submetidos a otoplastia, não obteve nenhum resultado negativo. Em nosso estudo, tivemos 4 resultados negativos (mínimo $=-16,67$ ) na área de saúde física, evidenciando uma piora nessa subárea desses pacientes nos primeiros 2 meses após a cirurgia. Hipóteses levantadas para este resultado foram a necessidade de irem para consultas pós-operatórias e a utilização de mais medicamentos, tais como antibióticos e analgésicos, após a cirurgia. Quatorze pacientes pontuaram zero na categoria saúde física da ERG, não evidenciando nem melhora nem piora neste setor específico.

Mesmo com resultados negativos e no valor de zero na subárea de saúde física (4 e 18 pacientes, respectivamente), o valor positivo da pontuação total da ERG foi significativo, corroborando o conceito da OMS de que saúde não é apenas a ausência de doença, que inclui também o bem-estar físico, mental e social. ${ }^{2}$

Não foram encontrados estudos que correlacionassem a Escala Visual Analógica com a Escala de Resultados de Glasgow. O presente estudo calculou o Delta EVA como forma de correlacioná-los. Comparando o Delta EVA com a ERG foi possível demonstrar que as medidas se mostraram significativamente correlacionadas na pontuação total da ERG e nas subáreas de saúde geral e relações sociais ( $\rho$ de Spearman de 0,$599 ; 0,571$ e 0,445 , respectivamente). Assim como não houve resultados positivos na área de saúde física da ERG, também não houve correlação significativa entre o Delta EVA e essa subárea. Dessa forma, evidenciou-se que a melhora na satisfação da aparência do paciente interfere diretamente na qualidade de vida.

O estudo não mostrou correlação significativa entre o Delta EVA e a idade ( $\rho$ de Spearman $=0,347$ e $p=0,077$ ), mas evidenciou relação significativa positiva entre a ERG e a idade ( $\rho$ de Spearman para a pontuação total de 0,437 e $p=0,022$ ). Uma das hipóteses para este resultado é o fato de pessoas mais velhas apresentarem mais anos de sofrimento psicológico, resultando em uma melhora maior na qualidade de vida. A 
idade não é uma variável de confusão na análise de associação de Delta EVA com ERG, pois os resultados estatísticos foram os mesmos após a análise de correlação parcial, sendo a idade a variável de controle.

Não houve nenhuma complicação pós-operatória grave. A maior dificuldade que encontramos no estudo foi frente ao não comparecimento dos pacientes no retorno após 2 meses de cirurgia, mesmo após tentativas de contato.

\section{REFERÊNCIAS}

1. Whoqol Group. Development of the WHOQOL: rationale and current status. Int J Ment Health. 1994;23:24-56.

2. Whoqol Group. The World Health Organization quality of life assessment (WHOQOL): development and general psychometric properties. Soc Sci Med. 1998;46:1569-85.

3. Fleck MP. O instrumento de avaliação de qualidade de vida da Organização Mundial da Saúde (WHOQOL-100): características e perspectivas. Cien Saude Colet. 2000;5:33-8.

4. Marone SA, Linhares TA Filho, Ishie RT, Dode OB, Faria BC, Rodrigues JL, et al. Using ERG inquiry to evoluate otoplasty satisfaction in an otorhinolaryngology medical residency training hospital. Braz J Otorhinolaryngol. 2012;78:113-9.

5. Songu M, Kutlu A. Health-related quality of life outcome of children with prominent ears after otoplasty. Eur Arch Otorhinolaryngol. 2014;271:1829-32.

6. Choi JY, Jung SC, Sykes JM. Clinical outcome and patients' satisfaction study after otoplasty using hybrid techniques in adult patients. J Craniofac Surg. 2017;28:1278-81.

7. Songu M, Kutlu A. Long-term psychosocial impact of otoplasty performed on children with prominent ears. J Laryngol Otol. 2014;128:768-71.

8. Mustardée JC. The treatment of prominent ears by buried mattress sutures. Plast Reconstr Surg. 1967;39:382-6.

9. Furnas DW. Correction of prominent ears by concha-mastoid sutures. Plast Reconstr Surg. 1968;42:189-94.

10. Maniglia AJ, Maniglia JJ, Witten BR. Otoplasty - an eclectic technique. Laryngoscope. 1977;87:1359-68.

\section{CONCLUSÃO}

A Otoplastia mostrou-se um fator importante de melhora na qualidade de vida de pacientes com orelhas proeminentes, principalmente na saúde geral e nas relações sociais desses pacientes. Através da comparação e da análise da EVA no pré e pós-operatório, Delta EVA e ERG, foi possível concluir que a melhora na satisfação com a aparência física é um fator preditor direto de mudança em suas qualidades de vidas.

11. Ribeiro FA, Bussoloti I Filho, Scalia RA, Corvo MA. Otoplastia. In: Pignatari SS, Anselmo-Lima WT, editors. Tratado de otorrinolaringologia. 3. ed. Rio de Janeiro: Elsevier; 2018. p. 870-80.

12. Papadopulos NA, Niehaus R, Keller E, Henrich G, Papadopulus ON, Staudenmaier R et al. The psychologic and psychosocial impact of otoplasty on children and adults. J Craniofac Surg. 2015;26:2309-14.

13. Brandão MP, Rebelo C. Uma revisão sistemática de instrumentos sobre qualidade de vida em pessoas idosas após implante coclear. Rev Port Saúde Pública. 2017;35:10-8.

14. Robinson K, Gatehouse S, Browning GG. Measuring patient benefit from otorhinolaryngological surgery and therapy. Ann Otol Rhinol Laryngol. 1996;105:415-22.

15. Robinson K, Gatehouse S, Browning GG. The glasgow health status questionnaires manual [Internet]. Glasgow, Scotland: MRC Institute of Hearing Research, Glasgow Royal Infirmary; 1998 [acesso em: 30 Set 2020]. Disponível em: https://scholar.google. $\mathrm{com} /$ scholar lookup?title $=$ The + Glasgow + Health + Status + Questionn aires+Manual\&publication_year=1998\&

16. Monini S, Bianchi A, Talamonti R, Atturo F, Filippi C, Barbara M. Patient satisfaction after auditory implant surgery: ten-year experience from a single implanting unit center. Acta Otolaryngol. 2016;137:389-97.

17. Harris PA, Taylor R, Thielke R, Payne J, Gonzalez N, Conde JG. Research electronic data capture (REDCap) - a metadata-driven methodology and workflow process for providing translational research informatics support. J Biomed Inform. 2009;42:377-81.

18. Braun T, Hainzinger T, Stelter K, Krause E, Berghaus A, Hempel JM. Health-related quality of life, patient benefit, and clinical outcome after otoplasty using suture techniques in 62 children and adults. Plast Reconstr Surg. 2010;126:2115-24.

\section{Como citar:}

Conceição MB, Matos AC, Almeida CM, Viana AB Júnior, Silva VC, Jorge RB, et al. Impacto na qualidade de vida de pacientes submetidos a otoplastia em um hospital universitário. Rev Med UFC. 2021;61(1):1-5. 